\title{
Shear Bond Strength of Several Dental Cements
}

\section{Afutu R*, Daddona J, Dunn K, Finkelman M, Tran A and Kugel G Tufts University School of Dental Medicine, Boston, USA}

*Corresponding author: Roberta Afutu, Tufts University School of Dental Medicine, Boston, MA, USA, Tel: 6176362115; Email: Roberta.Afutu@tufts.edu

\section{Research Article \\ Volume 4 Issue 3}

Received Date: July 20, 2019

Published Date: August 01, 2019

DOI: 10.23880 /oajds-16000234

\section{Abstract}

Purpose: To compare the shear bond strength (SBS) of various cements when bonded to zirconia, e.max crowns, titanium, stainless steel, and dentin using a universal testing machine.

Materials and Methods: Products tested were: Group 1: ACTIVA $^{\mathrm{TM}}$ BioACTIVE Cement without DenTASTIC $^{\mathrm{TM}}$ UNO $^{\mathrm{TM}}$ (Pulpdent) (AB), Group 2: Rely $X^{\mathrm{TM}}$ Unicem 2 Automix (3M) (RelyX), Group 3: Ceramir® Crown \& Bridge (Doxa) (CeraCB), Group 4: FujiCEM ${ }^{\text {TM }} 2$ Cement (GC America) (FCEM), and Group 5: ACTIVA BioACTIVE Cement with DenTASTIC UNO (Pulpdent) (ABD). The cement Groups 1-4 (n=25) were tested against five substrates, and ABD $(n=5)$ was tested against dentin and was not tested against the other four substrates. ABD used five caries-free human molar teeth were mounted in acrylic resin with the long axis of the tooth perpendicular to the surface and flattened using 240 grit silicon paper. Each cement was bonded to their appropriate substrate using cylindrical molds, with an internal diameter of $2.38 \mathrm{~mm}$ and height of $2 \mathrm{~mm}$. All samples were cured (Translux® Wave, Heraeus Kulzer) and placed in water at $37^{\circ} \mathrm{C}$ for 24 hours prior to testing to ensure complete polymerization of the material. A universal testing machine (Instron $\AA 5566 A$ ) was used to test the SBS with $1 \mathrm{~mm} / \mathrm{min}$ crosshead speed.

Results: AB showed statistically significant superior bonds for SBS when compared to CeraCB and FCEM for all five substrates, and only for zirconia when compared to RelyX. There was no statistical significant difference for SBS when comparing dentin bonding between $\mathrm{AB}$ and $\mathrm{ABD}$. RelyX showed statistically significant superior SBS when compared to CeraCB and FCEM for all substrates, except dentin. CeraCB and FCEM did not show any statistical significance except when bonded to e.max ( $\mathrm{p}=00079)$.

Conclusion: $\mathrm{AB}$ compared to $\mathrm{ABD}$ showed no significant difference in terms of dentin bonding. The addition of a primer to the original product did not produce additional adhesive cementation on dentin testing. However, $A B$ and $A B D$, showed better SBS for dentin compared to RelyX, CeraCB and FCEM. Based on this study, AB and RelyX appear to be appropriate cements for all substrates tested.

Keywords: Conventional glass ionomer cements; Resin modified glass ionomer cements; Shear bond strength; Material substrates; Dental cements 


\section{Open Access Journal of Dental Sciences}

Abbreviations: GI: Glass Ionomer; RGMI: Resin Modified Glass Ionomer; SBS: Shear Bond Strength.

\section{Introduction}

The most widely used dental cements in the United States fall into two categories, Conventional Glass Ionomer (GI) and Resin Modified Glass Ionomer (RGMI). While conventional GIs are classified as cements, they are most typically used as restorative materials. RMGIs can be used for restorative procedures as well as for the cementation of crowns. Both conventional GI and RMGIs are said to have excellent bond strengths and sealing in the short term; making clinicians' decisions difficult [1]. According to literature, formation of a micro-mechanical bond is believed to be a prerequisite to the accomplishment of a strong mechanical bond [1]. Additional chemical adhesion may be beneficial to the durability and longevity of a dental restoration as it ensures an intimate adaptation of both the tooth structure and cement components [2].

Ceramir Crown and Bridge and ACTIVA Bio-ACTIVE cements are classified as bioactive. This type of cement contains numerous oxides that are said to produce a strong bond through the "production of hydroxyapatite and the formation of a strong bond between collagen and the hydroxyapatite" [3]. ACTIVA contains three key components, bioactive ionic resin matric, shockabsorbing rubberized resin, and reactive ionomer glass fillers [3]. Ceramir Crown and Bridge is not a resin cement, but contains the physical properties that equal or exceed resin-based, RMGI and adhesive resin cements [4]. The bioactivity of ACTIVA stimulates the natural remineralization process by continuously forming mineral apatite crystals which form ionic bonds. This continuously forming bond is responsible for reducing marginal gaps and reducing microleakage which protects against recurrent caries and failure of the restoration [3].

RelyX Unicem 2 is an RMGI cement. It delivers bond strength for porcelain-fused-to-metal crowns and bridges, metal crowns, inlays and onlays, and ceramic restorations [5]. This non- bioactive cement does not re-mineralize tooth structure. FujiCEM 2 is a second-generation RMGI. Its F2 Flex Fuse Technology incorporates high-elastic crosslinking monomers that increase strength properties; helping to improve indirect restorations with increased strength, high fluoride release, low film thickness, and exceptional marginal integrity [6]. This cement is indicated for a broad array of all types of metal-, resin-, and zirconia-based inlays, outlays and crowns and bridges [6].
Throughout this study, we analyzed the efficiency of different dental cements when bonded to a variety of popular restoration substrates. Zirconia in dentistry has expanded the possible applications of metal-free ceramic restorations with greater success and reliability [7]. Zirconia holds strong optical and mechanical properties [7]. Its surface stability generates efficiency of the chemical or mechanical bond [7]. Lithium disilicate is a glass-ceramic that is compatible with either adhesive, self-adhesive or conventional cementation, depending on the indication [8]. Titanium and other metals such as, stainless steel are artificial materials lack biofunction [9]. Surface modification is necessary to change the materials composition to promote a strong bond.

The aim of this study is to analyze the mechanical strength of resin modified glass ionomers and conventional glass ionomers used clinically for restorations or seating restorations. Shear bond strength performed to test adhesion strength to different clinically used substrates including ceramic material, metal and dentin surfaces.

\section{Materials and Methods}

\section{Sample Preparation}

Each of the five substrate groups were mounted in acrylic. Blue infection control tape was placed on the bottom of a model holder to serve as a base. The acrylic resin was poured into a model holder that is $25 \mathrm{~mm}$ deep and $25 \mathrm{~mm}$ in diameter. e. Zirconia, IPA max crowns (Ivoclar), Ti-6Al-4V Titanium, and stainless steel substrates were embedded in the acrylic exposing 1 flat surface to bond each button $(2.38 \mathrm{~mm} X 2.00 \mathrm{~mm})$ of cement. The fifth group, dentin, contained 5 caries-free human molar teeth that were stored in a 1:10 solution of hydrochloric acid and distilled water. The dentin samples were then mounted in an acrylic resin with the long axis of the tooth perpendicular to the surface and flattened to expose the clinical dentin using 240 and 600 grit silicon paper.

A cement button was bonded to each substrate using an Ultradent jig (Figures $1 \& 2$ ) according to manufacturers' instructions. Products tested were:

- Group 1: ACTIVA $^{\mathrm{TM}}$ BioACTIVE Cement without DenTASTIC ${ }^{\mathrm{TM}} \mathrm{UNO}^{\mathrm{TM}}(\mathrm{AB})$,

- Group 2: Rely $\mathrm{X}^{\mathrm{TM}}$ Unicem 2 Automix (RelyX),

- Group 3: Ceramir® Crown \& Bridge (CeraCB),

- Group 4: FujiCEM ${ }^{\mathrm{TM}} 2$ Cement (FCEM), and

- Group 5: ACTIVA BioACTIVE Cement with DenTASTIC UNO (ABD). 
The cement Groups 1-4 $(n=25)$ were tested against five substrates, and $A B D(n=5)$ was tested against dentin and was not tested against the other four substrates.

$\mathrm{AB}$ was allowed to self-cure for 3 minutes followed by a 20 second light cure (Translux® Wave, Heraeus Kulzer). RelyX was allowed to self-cure for 3 minutes following by a 20 second light cure (Translux Wave, Heraeus Kulzer) on every $4 \mathrm{~mm}$ span. CeraCB was allowed to self- cure for 5 minutes. FCEM was allowed to self-cure for 4 minutes and 30 seconds. ABD's slightly moist dentin was coated with DenTASTIC UNO and light cured (Translux Wave, Heraeus Kulzer) for 10 seconds. The cement was then applied and light cured (Translux Wave, Heraeus Kulzer) for 20 seconds. After the bonding procedure was completed, all samples were stored in deionized water at $37^{\circ} \mathrm{C}$ for an additional 24 hours prior to testing to ensure complete polymerization of the material.

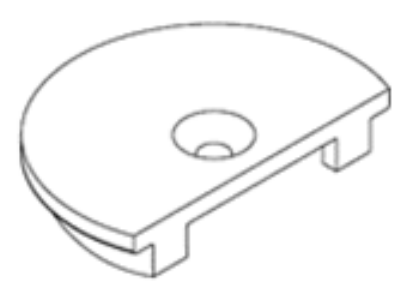

Figure 1: Button mold insert used for cement bonding.

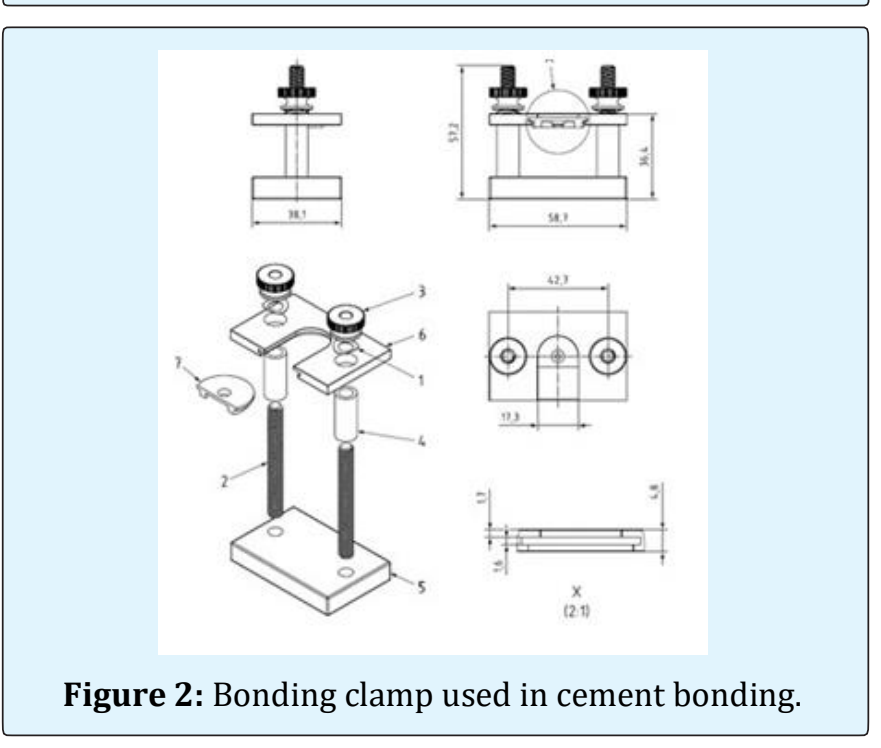

\section{Shear Bond Strength Testing}

The Instron® 5566A (Norwood, MA) was used to test the bond strength of each group with a $1 \mathrm{~mm} / \mathrm{min}$ notched-edge crosshead speed (Figure 3).

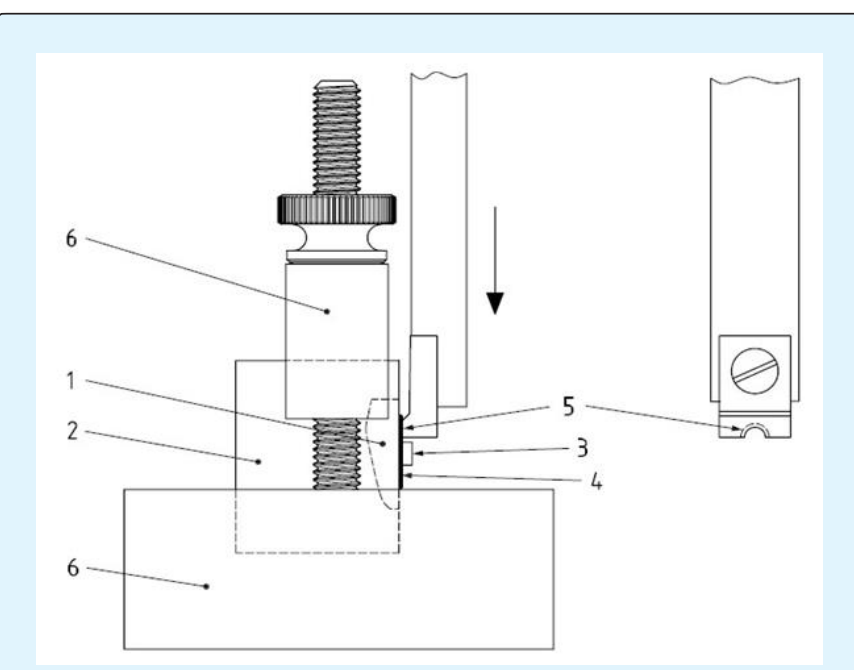

Key: 1. Tooth; 2. Cured potting material; 3.Cured composite button; 4 . Cured adhesive; 5 . Notched-Edge shear blade; 6 . Test base clamp.

Figure 3: Bonding sample in test base clamp with notched-edge crosshead.

\section{Results}

The difference in shear bond strength was statistically significant on all substrates with each cement tested. AB showed statistically significant superior bonds for shear bond strength when compared to CeraCB and FCEM for all five substrates, and only for zirconia when compared to RelyX. There was no statistical significant difference for shear bond strength when comparing dentin bonding between $\mathrm{AB}$ and $\mathrm{ABD}$. RelyX showed statistically significant superior shear bond strength when compared to CeraCB and FCEM for all substrates, except dentin.

ABD showed statistically superior shear bond strength to dentin when compared to RelyX, CeraCB and FCEM. FCEM did not show any statistical significance except when bonded to e.max ( $\mathrm{p}=00079)$ (Tables $1 \& 2)$, (Figure 4).

\begin{tabular}{|c|c|}
\hline Abbreviation & Cement/Manufacturer \\
\hline $\mathrm{AB}$ & $\begin{array}{l}\text { ACTIVA }^{\text {TM }} \text { BioACTIVE Cement without } \\
\text { DenTASTICTM }^{\text {TNO }} \text { UNM }^{\text {TM }} \text { (Pulpdent) }\end{array}$ \\
\hline Rely X & Rely $\mathrm{X}^{\mathrm{TM}}$ Unicem 2 Automix (3M) \\
\hline CeraCB & Ceramir ${ }^{\circ}$ Crown \& Bridge (Doxa) \\
\hline FCEM & FujiCEM $^{\mathrm{TM}} 2$ Cement (GC America) \\
\hline $\mathrm{ABD}$ & $\begin{array}{l}\text { ACTIVA BioACTIVE Cement with } \\
\text { DenTASTIC UNO (Pulpdent) }\end{array}$ \\
\hline
\end{tabular}

Table 1: Abbreviations for the tested GI and RGMI cements. 


\section{Open Access Journal of Dental Sciences}

\begin{tabular}{|c|c|c|c|c|c|c|c|c|c|c|}
\hline Substrate & \multicolumn{2}{|c|}{ AB } & \multicolumn{2}{c|}{ RelyX } & \multicolumn{2}{c|}{ CeraCB } & \multicolumn{2}{c|}{ FCEM } & \multicolumn{2}{c|}{ ABD } \\
\hline Zirconia & 12.8 & \pm 3.5 & 14.6 & \pm 3.1 & 0 & \pm 0 & 2.4 & \pm 3.5 & - & - \\
\hline IPA e.max Crowns & 14.1 & \pm 1.7 & 19.5 & \pm 0.9 & 0 & \pm 0 & 3.1 & \pm 1.6 & - & - \\
\hline Titanium & 4.6 & \pm 1.3 & 5.5 & \pm 2.0 & 0 & \pm 0 & 0.3 & \pm 0.5 & - & - \\
\hline Stainless Steel & 2 & \pm 0.8 & 2.8 & \pm 0.8 & 0 & \pm 0 & 0 & \pm 0 & - & - \\
\hline Dentin & 12.5 & \pm 2.1 & 3.6 & \pm 0.5 & 2.8 & \pm 2.2 & 2 & \pm 2.5 & 11.9 & \pm 2.6 \\
\hline
\end{tabular}

Table 2: Shear bond strength of several dental cements.

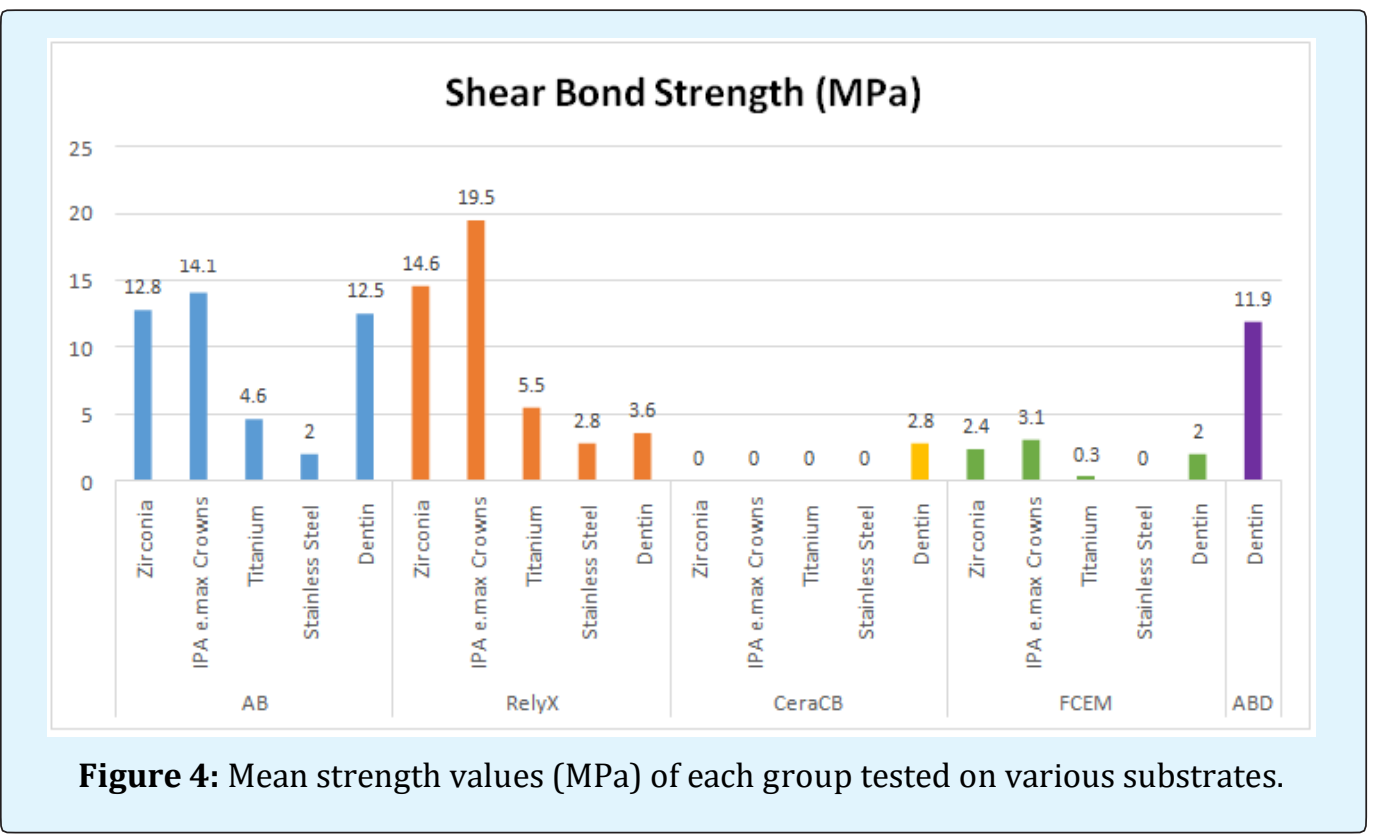

\section{Discussion}

$\mathrm{AB}$ contains hydrophilic properties where it extracts fluoride, calcium and phosphate from the saliva and releases these ions to the tooth [10]. The manufacturer claims that $A B$ demonstrates intimate adaptation to the tooth structure. The chemical bonding that takes place between the tooth and the material creates durability and fracture resistance [10]. The changing $\mathrm{pH}$ qualities of the material allow the physical properties of the tooth structure to stimulate minor regeneration [10]. $A B$ resembles physical qualities of glass ionomer systems and traditional composite resin chemistry [10].

ABD showed statistically superior shear bond strength for the majority of the substrates when compared to RelyX, CeraCB and FCEM. RelyX showed statistically superior shear bond strength for all the substrates when compared to CeraCB and FCEM, except dentin. AB and RelyX showed comparably similar shear bond strength for all substrates tested except zirconia and dentin; Rely X may be an appropriate substitution for $\mathrm{AB}$.
$A B$ compared to ABD showed no significant difference in terms of dentin bonding. The addition of the DenTASTIC UNO and bonding agent to the original product did not produce a more adhesive cementation on dentin testing. This leads to the possibility that it may not be needed, however, further research is needed to confirm. $\mathrm{AB}$ and $\mathrm{ABD}$ showed better shear bond strength for dentin compared to the other three products; RelyX, CeraCB and FCEM.

\section{Conclusion}

Within in the limitations of this in vitro study, it was concluded that cements, $\mathrm{AB}$ and $\mathrm{ABD}$, on exposed dentin surface has an influence on bond strength values. The clinic relevance is to examine the bond strength of between several cements on a wide range of substrates.

Adding DenTASTIC UNO did not significantly increase the bond of $\mathrm{AB}$ to the dentin surface. Bond strength of the dentin substrates for groups RelyX, CeraCB and FCEM was significantly lower than $\mathrm{AB}$ and $\mathrm{ABD}$. However, RelyX applied to the IPA e.max crown substrate had a higher 
bond strength than $\mathrm{AB}$. Based on the findings of this study, $\mathrm{ABD}$ appears to be the permanent cementation of any restoration type tested. The bioactivity of $A B$ is responsible for continuously forming ionic bond that protects against recurrent caries and failure of the restoration, leading to its overall better longevity and durability.

\section{Regulatory Statement}

This study was conducted in accordance with all the provisions of the International Review Board's oversight committee guidelines and policies of Tufts University School of Dental Medicine in Boston, MA.

\section{Conflict of Interest}

This study was sponsored in part by Pulpdent Corporation.

\section{References}

1. De Munick J, Van Landuyt K, Peumans M, Poitevin A, Lambrechts P (2005) A critical review of the durability of adhesion to tooth tissue: methods and results. J Dent Res 84(2): 118-132.

2. Sano H (1999) Long-term durability of dentin bonds made with self-etching primer, in vivo. J Dent Res 78(4): 906-911.
3. (2017) ACTIVA BioActive White Paper. Pulpdent http://www.pulpdent.com/?_id2932

4. (2017) Facts about Ceramir Crown \& Bridge. Ceramir http://ceramirus.com/wp-

content/uploads/Ceramir-Technical-Product-

Profile.pdf

5. (2017) RelyX Luting Plus Brochure 3M. http://mutlimedia.3m.com/mws/media/8691530/re lyx-luting-plus-brochure.pdf

6. (2017) GC FujiCEM 2 Brochure, GC America.

7. Gargari M, Gloria F, Napoli E, Pujia AM (2010) Zirconia: Cementation of Prosthetic Restorations, Literature Review. Oral Implantology 3(4): 25-29.

8. (2019) IPS e.max® CAD Brochure”. Ivoclar Vivadent AG. http://downloads.ivoclarvivadent.com/zooluwebsite/media/document/1255/IPS+e max+CAD++ Lab+to+Dentist

9. Hanawa $\mathrm{T}$ (2011) A comprehensive review of techniques for biofunctionalization of titanium. J Periodontal Implant Sci 41(6): 263-272.

10. Owens BM, Phebus JG, Johnson WW (2018) Evaluation of the marginal integrity of a bioactive restorative material. Gen Dent 66(3): 32-36. 